\title{
ON LIPSCHITZ-LIKE CONTINUITY OF A CLASS OF SET-VALUED MAPPINGS
}

\author{
EWA M. BEDNARCZUK ${ }^{1}$, LEONID I. MINCHENKO ${ }^{2}$, AND KRZYSZTOF E. RUTKOWSKI ${ }^{3}$
}

\begin{abstract}
We study set-valued mappings defined by solution sets of parametric systems of equalities and inequalities. We prove Lipschitz-like continuity of these mappings under relaxed constant rank constraint qualification.
\end{abstract}

\section{INTRODUCTION}

Properties of set-valued mappings given by systems of equalities and inequalities play a significant role in parametric optimization. In particular, considerable effort is directed towards formulating conditions ensuring Lipschitz-type continuities of these mappings, namely their calmness and the pseudo-Lipschitz continuity (also referred to as Lipschitz-like continuity or the Aubin property) $[1,13,18,19,24]$.

The present paper is devoted to sufficient conditions for R-regularity (Definition 2.5) and pseudo-Lipschitz continuity for set-valued mappings defined by solution sets of parametric constrained systems. R-regularity is a variant of a much more general property, called metric regularity, intensively studied in $[8,9,11,16,17,21]$.

Let $\mathcal{H}$ be a Hilbert space and $\mathcal{G}$ be a normed space. Let us consider a parametric nonlinear programming problem:

$$
\begin{array}{ll}
\text { minimize } & f(p, x) \\
\text { subject to } & x \in F(p)=\left\{x \in \mathcal{H} \mid h_{i}(p, x) \leq 0, i \in I, h_{i}(p, x)=0, i \in I_{0}\right\},
\end{array}
$$

where $p \in \mathcal{G}$ is a parameter, $x \in \mathcal{H}$ stands for the decision variable, $I=\{1, \ldots, m\}$, $I_{0}=\{m+1, \ldots, n\}$ (we admit the case $I_{0}=\emptyset$ ). Functions $f, h_{i}: \mathcal{G} \times \mathcal{H} \rightarrow \mathbb{R}$, $i=1, \ldots, n$, are assumed to be (jointly) continuous together with their partial gradients with respect to $x, \nabla_{x} f$ and $\nabla_{x} h_{i}, i=1, \ldots, n$.

In the present paper we prove Lipschitz-likeness of the set-valued mapping $F$ defined in (1.1). We generalize results from [25] and [3]. In [25] the respective results are obtained under stronger assumptions of functions $h_{i}, i \in I_{0} \cup I$, while in [3] the Lipschitz-likeness of $F$ is obtained for $h_{i}(p, x)=\left\langle x \mid g_{i}(p)\right\rangle-f_{i}(p), i \in I_{0} \cup I$, where $f_{i}: \mathcal{G} \rightarrow \mathbb{R}, g_{i}: \mathcal{G} \rightarrow \mathcal{H}, i \in I_{0} \cup I$, are locally Lipschitz functions. We also correct the mistake in the proof of Lemma 3 of [25].

2010 Mathematics Subject Classification. 41A50, 46C05, 49K27, 52A07, 90C31.

Key words and phrases. set-valued mappings, parametric optimization, relaxed constant rank constraint qualification, R-regularity, pseudo-Lipschitz continuity, Lipschitz-like continuity, Aubin property.

${ }^{1}$ Systems Research Institute of the Polish Academy of Sciences, Warsaw University of Technology.

2 Belarus State University of Informatics and Radioelectronics, Minsk, Belarus .

${ }^{3}$ Warsaw University of Technology, Systems Research Institute of the Polish Academy of Sciences 
For set-valued mapping $F: \mathcal{G} \rightrightarrows \mathcal{H}$ defined in (1.1), its domain and graph are defined by $\operatorname{dom} F=\{p \in \mathcal{G} \mid F(p) \neq \emptyset\}$ and $g r F=\{(p, x) \mid x \in F(p), p \in \mathcal{G}\}$, respectively.

The tangent cone $T(F(p), x)$ and the linearized cone $\Gamma(F(p), x)$ to $F(p)$ at $x \in F(p)$ are defined, respectively, as follows

$T(F(p), x)=\left\{d \in \mathcal{H} \mid \exists t_{k} \downarrow 0, d^{k} \rightarrow d\right.$ such that $\left.x+t_{k} d^{k} \in F(p), k=1,2, \ldots\right\}$,

$\Gamma(F(p), x)=\left\{d \in \mathcal{H} \mid\left\langle\nabla_{x} h_{i}(p, x), d\right\rangle \leq 0: i \in I(p, x),\left\langle\nabla_{x} h_{i}(p, x), d\right\rangle=0: i \in I_{0}\right\}$.

Organization of the paper is as follows. In section 2 we provide basing concepts. Section 3 is devoted to the concept of $R$-regularity. In section 4 we investigate relationships between relaxed constant rank condition and the R-regularity of $F$. In section 5 we prove Lipschitz-likeness of $F$ under relaxed constant rank condition. In section 6 some applications to bilevel programming are discussed.

\section{BASIC CONCEPTS AND DEFINITIONS}

This section contains some background material (see, i.e., $[1,15,19,22,24,26]$ ) which will be used in the sequel.

We denote $V_{\delta}\left(p^{0}\right)=p^{0}+\delta B, V_{\varepsilon}\left(x^{0}\right)=x^{0}+\varepsilon B$, where $B$ is the open unit ball centered at 0 in the respective space, $\operatorname{dist}(v, C):=\inf \{\|v-c\|, c \in C\}$ is the distance between point $v$ and set $C$, where $\|v\|$ is the norm of vector $v$.

Definition 2.1. A set-valued mapping $F$ is lower semicontinuous (Isc) at $\left(p^{0}, x^{0}\right) \in$ grF (relative to $P \subset \mathcal{G}$ ) if for any neighbourhood $V\left(x^{0}\right)$ there is a neighborhood $V\left(p^{0}\right)$ such that $F(p) \cap V\left(x^{0}\right) \neq \emptyset$ for all $p \in V\left(p^{0}\right)$ (for all $p \in V\left(p^{0}\right) \cap P$ ).

Definition 2.2. A set-valued mapping $F$ is lower Lipschitz continuous at $\left(p^{0}, x^{0}\right) \in \operatorname{gr} F$ (relative to $P \subset \mathcal{G}$ ) if there exist positive numbers $l$ and $\delta$ such that

$$
\operatorname{dist}\left(x^{0}, F(p)\right) \leq l\left\|p-p^{0}\right\| \quad \forall p \in V_{\delta}\left(p^{0}\right) \quad\left(\forall p \in V_{\delta}\left(p^{0}\right) \cap P\right) .
$$

Note that (2.1) implies that $F(p) \cap V_{\varepsilon}\left(x^{0}\right) \neq \emptyset$ for any $\varepsilon>0$, if $\left\|p-p^{0}\right\|<$ $\min \{\delta, \varepsilon / l\}$.

Definition 2.3. A set-valued mapping $F$ is Lipschitz-like (pseudo-Lipschitzian) (relative to $P \subset \mathcal{G}$ ) at $\left(p^{0}, x^{0}\right) \in \operatorname{grF}$ (where $p^{0} \in P$ ) if there exist a number $l_{F}>0$ and neighbourhoods $V\left(p^{0}\right)$ and $V\left(x^{0}\right)$ such that

$$
F\left(p^{1}\right) \cap V\left(x^{0}\right) \subset F\left(p^{2}\right)+l_{F}\left\|p^{2}-p^{1}\right\| B
$$

for all $p^{1}, p^{2} \in V\left(p^{0}\right)\left(p^{1}, p^{2} \in V\left(p^{0}\right) \cap P\right)$.

Let $P \subset \mathcal{G}, X \subset \mathcal{H}$ and $I(p, x):=\left\{i \in I \mid h_{i}(p, x)=0\right\}$ be the set of indices of active inequality constraints at $(p, x) \in g r F$. Following [24, 25] we define the relaxed constant rank constraint qualification (RCRCQ) which generalizes the constant rank constraint qualification introduced by Janin [18].

Definition 2.4. The set-valued mapping $F$ satisfies the Relaxed Constant Rank Constraint Qualification, or shortly, RCRCQ (relative to $P \times X)$ at $\left(p^{0}, x^{0}\right) \in g r F$, if for any index set $K \subset I\left(p^{0}, x^{0}\right)$

$$
\operatorname{rank}\left\{\nabla_{x} h_{i}(p, x): i \in I_{0} \cup K\right\}=\operatorname{rank}\left\{\nabla_{x} h_{i}\left(p^{0}, x^{0}\right): i \in I_{0} \cup K\right\}
$$

in a neighbourhood of $\left(p^{0}, x^{0}\right)$ (for $(p, x) \in P \times X$ from this neighbourhood). 
The set $F\left(p^{0}\right)$ satisfies $R C R C Q$ at $x^{0} \in F\left(p^{0}\right)$ if for any index set $K \subset I\left(p^{0}, x^{0}\right)$

$$
\operatorname{rank}\left\{\nabla_{x} h_{i}\left(p^{0}, x\right): i \in I_{0} \cup K\right\}=\operatorname{rank}\left\{\nabla_{x} h_{i}\left(p^{0}, x^{0}\right): i \in I_{0} \cup K\right\}
$$

for all $x$ in a neighbourhood of $x^{0}$.

Clearly, if the set-valued mapping $F$ satisfies RCRCQ at $\left(p^{0}, x^{0}\right) \in \operatorname{gr} F$, then it satisfies RCRCQ at all points $(p, x) \in g r F$ in some neighbourhood of $\left(p^{0}, x^{0}\right)$.

The following lemma proves the equality $\Gamma(F(p), x)=T(F(p), x)$ under RCRCQ. In the finite dimensional case, where $\mathcal{H}=\mathbb{R}^{s}$ this fact has been proved in Theorem 1 of [24]. In the infinite-dimensional case considered in the present paper this fact has been proved in Theorem 6.3 of [2].

Lemma 2.1. ([2, 24]). Let the set-valued mapping $F$ satisfy $R C R C Q$ (relative to $\operatorname{dom} F \times \mathcal{H})$ at $\left(p^{0}, x^{0}\right) \in \operatorname{gr} F$. Then there exist neighborhoods $V\left(p^{0}\right)$ and $V\left(x^{0}\right)$ such that $\Gamma(F(p), x)=T(F(p), x)$ for all $x \in F(p) \cap V\left(x^{0}\right)$ and all $p \in V\left(p^{0}\right) \cap \operatorname{dom} F$.

Proof. As already noted, if $F$ satisfies RCRCQ at $\left(p^{0}, x^{0}\right)$, there are neighbourhoods $V\left(p^{0}\right)$ and $V\left(x^{0}\right)$ such that $F$ satisfies RCRCQ at any point $(p, x)$ where $p \in V\left(p^{0}\right) \cap$ $\operatorname{dom} F, x \in F(p) \cap V\left(x^{0}\right)$. Hence, the set $F(p)$ satisfies RCRCQ at $x$ and by Theorem 6.3 of [2], $\Gamma(F(p), x)=T(F(p), x)$.

Following $[12,22]$ we define the R-regularity of set-valued mappings.

Definition 2.5. The set-valued mapping $F$ is R-regular at $\left(p^{0}, x^{0}\right) \in \operatorname{gr} F$ (relative to $P \subset \mathcal{G})$ if there exist a number $M>0$ and neighbourhoods $V\left(p^{0}\right)$ and $V\left(x^{0}\right)$ such that

$$
\begin{aligned}
& \operatorname{dist}(x, F(p)) \leq M \max \left\{0, h_{i}(p, x), i \in I,\left|h_{i}(p, x)\right|, i \in I_{0}\right\} \\
& \text { for all } x \in V\left(x^{0}\right) \text { and } p \in V\left(p^{0}\right) \quad\left(p \in V\left(p^{0}\right) \cap P\right) .
\end{aligned}
$$

The concept of R-regularity appears in different works (see e.g. Theorem 2.84 and formula (2.164) of [4], formula (10) of [10]). When $h_{i}(x, p)=g_{i}(x)-p_{i}, g_{i}(p)$ : $\mathcal{G} \rightarrow \mathbb{R}, p_{i} \in \mathbb{R} i \in I \cup I_{0}$ the R-regularity is equivalent to the metric regularity of $G(x, p):=\left[g_{i}(x)-p_{i}\right]_{i \in I \cup I_{0}}-K$, where $K=\mathbb{R}_{-}^{m} \times\{0\}^{n-m}$ (see formulas (2.143), (2.144) of [4]). In the paper [20], some variants of (2.5) have been investigated (see e.g. formula (6) of [20]).

\section{Criterion of R-Regularity}

Let $v \in \mathcal{H}$ and $\Pi_{F(p)}(v):=\{\tilde{v} \in F(p) \mid\|\tilde{v}-v\|=\operatorname{dist}(v, F(p))\}$. The set $\Pi_{F(p)}(v)$ is the solution set to the problem

$$
\begin{array}{ll}
\text { minimize } & f_{v}(p, x)=\|x-v\| \\
\text { subject to } & x \in F(p) .
\end{array}
$$

The problem (3.1) can be equivalently reformulated as

$$
\begin{array}{ll}
\text { minimize } & \frac{1}{2}\|x-v\|^{2} \\
\text { subject to } & x \in F(p) .
\end{array}
$$

Lagrange multiplier sets for problem (3.1) are defined as follows

$$
\begin{aligned}
& \Lambda_{v}(p, x):=\left\{\lambda \in \mathbb{R}^{n} \mid \frac{x-v}{\|x-v\|}+\sum_{i=1}^{n} \lambda_{i} \nabla_{x} h_{i}(p, x)=0, \lambda_{i} \geq 0, \lambda_{i} h_{i}(p, u)=0 \text { for } i \in I\right\}, \\
& \Lambda_{v}^{M}(p, x):=\left\{\lambda \in \Lambda_{v}(p, x)\left|\sum_{i=1}^{n}\right| \lambda_{i} \mid \leq M\right\} .
\end{aligned}
$$


Lemma 3.1. (Proposition 7.1 of [2], Theorem 1 of [24]). Suppose that $x^{0} \in \mathcal{H}$ is a solution to (3.1) for $p=p^{0} \in \mathcal{G}$ and $v \in \mathcal{H}$. Assume the set-valued mapping $F$ satisfies RCRCQ (relative to $\operatorname{dom} F \times \mathcal{H})$ at $\left(p^{0}, x^{0}\right) \in \operatorname{grF}$. Then $\Lambda_{v}\left(p^{0}, x^{0}\right) \neq \emptyset$.

The following theorem generalizes Theorem 2 [25] and Theorem 4.1 [14] to parametric systems defined by the set-valued mapping $F$.

Theorem 3.1. Let $\left(p^{0}, x^{0}\right) \in g r F$ and the set-valued mapping $F$ be I.s.c. at $\left(p^{0}, x^{0}\right)$ relative to $\operatorname{dom} F$. The following assertions are equivalent:

(a) the set-valued mapping $F$ is $R$-regular at $\left(p^{0}, x^{0}\right)$ relative to domF;

(b) there exists a number $M>0$ such that for any sequences $p^{k} \rightarrow p^{0}, p^{k} \in$ $\operatorname{dom} F, v^{k} \rightarrow x^{0}, v^{k} \notin F\left(p^{k}\right)$, the inequality $\Lambda_{v_{k}}^{M}\left(p^{k}, x^{k}\right) \neq \emptyset$ holds for all $x^{k}=x\left(p^{k}, v^{k}\right) \in \Pi_{F\left(p^{k}\right)}\left(v^{k}\right)$ and $k$ sufficiently large.

Proof. If $x^{0} \in \operatorname{int} F\left(p^{0}\right)$, the theorem is obviously valid. Assume that $x^{0} \in b d F\left(p^{0}\right)$.

1) The implication $(a) \Rightarrow(b)$ follows from a slight modification in the first part of the proof of Theorem 2 [25].

2) $(b) \Rightarrow(a)$. On the contrary, suppose that $F$ is not R-regular relative $\operatorname{dom} F$ at $\left(p^{0}, x^{0}\right)$. Then there exist sequences $p^{k} \rightarrow p^{0}, p^{k} \in d o m F$, and $v^{k} \rightarrow x^{0}, v^{k} \notin$ $F\left(p^{k}\right)$, such that for all $k=1,2, \ldots$

$\operatorname{dist}\left(v^{k}, F\left(p^{k}\right)\right)>k \max \left\{0, h_{i}\left(p^{k}, v^{k}\right), i \in I,\left|h_{i}\left(p^{k}, v^{k}\right)\right|, i \in I_{0}\right\}$.

Take any $x^{k} \in \Pi_{F\left(p^{k}\right)}\left(v^{k}\right)$. Due to (b) there exists a vector $\lambda^{k}$ such that $\sum_{i=1}^{n}\left|\lambda_{i}^{k}\right| \leq M$ and

$$
\begin{aligned}
& \frac{x^{k}-v^{k}}{\left\|x^{k}-v^{k}\right\|}+\sum_{i=1}^{n} \lambda_{i}^{k} \nabla_{x} h_{i}\left(p^{k}, x^{k}\right)=0, \lambda_{i}^{k} \nabla_{x} h_{i}\left(p^{k}, x^{k}\right)=0, \\
& \lambda_{i}^{k} \geq 0 i \in I\left(p^{k}, x^{k}\right), \lambda_{i}^{k}=0 i \in I \backslash I\left(p^{k}, x^{k}\right) .
\end{aligned}
$$

It follows from the lower semicontinuity of $F$ at $\left(p^{0}, x^{0}\right)$ that there exists a sequence $q^{k} \in F\left(p^{k}\right)$ such that $q^{k} \rightarrow x^{0}$. Then $\left\|v^{k}-x^{k}\right\| \leq\left\|v^{k}-q^{k}\right\|$ and, therefore, $x^{k} \rightarrow x^{0}$.

In virtue of the boundedness of the sequence $\lambda^{k}$ and of the condition $\lambda_{i}^{k} h_{i}\left(p^{k}, x^{k}\right)=0$, for $i \in I$, by (3.4), for all sufficiently large $k$ we obtain

$$
\begin{aligned}
& \left\|x^{k}-v^{k}\right\|=\left\langle\sum_{i=1}^{n} \lambda_{i}^{k} \nabla_{x} h_{i}\left(p^{k}, x^{k}\right), v^{k}-x^{k}\right\rangle \\
& \leq \sum_{i=1}^{n} \lambda_{i}^{k}\left(h_{i}\left(p^{k}, v^{k}\right)-h_{i}\left(p^{k}, x^{k}\right)+o\left(\left\|v^{k}-x^{k}\right\|\right)\right)= \\
& \left.=\sum_{i=1}^{n} \lambda_{i}^{k} h_{i}\left(p^{k}, v^{k}\right)+\sum_{i=1}^{n} \lambda_{i}^{k} o\left(\left\|v^{k}-x^{k}\right\|\right)\right) \leq \sum_{i=1}^{n} \lambda_{i}^{k} h_{i}\left(p^{k}, v^{k}\right)+\frac{1}{2}\left\|v^{k}-x^{k}\right\| .
\end{aligned}
$$

The latter inequality implies

$$
\operatorname{dist}\left(v^{k}, F\left(p^{k}\right)\right)=\left\|x^{k}-v^{k}\right\| \leq 2 M \max \left\{0, h_{i}\left(p^{k}, v^{k}\right), i \in I,\left|h_{i}\left(p^{k}, v^{k}\right)\right|, i \in I_{0}\right\},
$$
which contradicts (3.3). Thus $(b) \Rightarrow(a)$.

Remark 3.2. Theorem 3.1 is a generalization of Theorem 2 [25]. Unlike Theorem 2 [25] it does not require Lipschitz continuity of gradients $\nabla_{x} h_{i}(p, x)$ for $i=1, \ldots, n$. Theorem 3.1 considers also more general notion of $R$-regularity relative $\operatorname{dom} F$. 
Remark 3.3. As follows from the proof of Theorem 2 [25] the implication $(a) \Rightarrow(b)$ holds without the assumptions of lower semicontinuity of $F$ at $\left(p^{0}, x^{0}\right)$.

Remark 3.4. Let us note that Lemma 3 of [25] is a consequence of Theorem 3.1 and Theorem (4.1) below, which says that RCRCQ for $F$ at $\left(p^{0}, x^{0}\right)$ and lower Lipschitz continuiuty of $F$ at $\left(p^{0}, x^{0}\right)$ implies $R$-regularity of $F$ at $\left(p^{0}, x^{0}\right)$.

In [3] we discussed in details the proof of Lemma 3 of [25] for some special functions $h_{i}, i=1, \ldots, n$. The proof of Theorem 3.1 together with Theorem 4.1 fill some gaps in the proof of Lemma 3 of [25].

The example below shows that the assertion of Theorem 3.1 may not hold if $F$ is not Isc at a point $\left(p^{0}, x^{0}\right)$.

Example 3.5. Let $F(p)=\left\{x \in \mathbb{R}^{2}\left|\left\|x_{1}\right\| \leq 1,\left\|x_{2}\right\| \leq 1, x_{2}-p x_{1}+\right| p \mid+1 \leq 0\right\}$, $p \in \mathbb{R}$. Then $F(p)=\{(1,-1)\}$ for all $p>0, F(p)=\{(-1,-1)\}$ for $p<0$ and $F(p)=\left\{x \in \mathbb{R}^{2} \mid-1 \leq x_{1} \leq 1, x_{2}=-1\right\}$ for $p=0$. Consider the point $\left(p^{0}, x^{0}\right)$, where $p^{0}=0, x^{0}=(0,-1)$.

Evidently, $F$ is not Isc at $\left(p^{0}, x^{0}\right)$. Let us take $p=\varepsilon>0, v=(\varepsilon,-1)$, where $\varepsilon \rightarrow 0$. It is easy to see that for given $v$ and $p$ the $R$-regularity condition does not hold if $\varepsilon$ is sufficiently small.

The following technical observation will be used in the sequel.

Proposition 3.6. Let $p^{0} \in \mathcal{D}$. Assume that $R C R C Q$ holds for the set-valued mapping $F$ given by (1.1) at $\left(p^{0}, x^{0}\right) \in$ gph $F$ and $F(p) \neq \emptyset$ for $p \in V_{0}\left(p^{0}\right)$. Then there exist neighbourhoods $V\left(p^{0}\right), V\left(x^{0}\right)$ and an index set $I_{0}^{\prime} \subset I_{0},\left|I_{0}^{\prime}\right|=\operatorname{rank}\left\{\nabla_{x} h_{i}\left(p_{0}, x_{0}\right) i \in\right.$ $\left.I_{0}\right\}$ such that for all $(p, x) \in V\left(p^{0}\right) \times V\left(x^{0}\right)$ vectors $\nabla_{x} h_{i}(p, x), i \in I_{0}^{\prime}$ are linearly independent.

Proof. The assertion is valid if $\nabla_{x} h_{i}\left(p^{0}, x^{0}\right), i \in I_{0}$ are linearly independent. Suppose that $\nabla_{x} h_{i}\left(p_{0}, x_{0}\right), i \in I_{0}$ are linearly dependent. By RCRCQ there exist neighbourhoods $V_{0}\left(p^{0}\right), V_{0}\left(x^{0}\right)$ such that

$$
\operatorname{rank}\left\{\nabla_{x} h_{i}(p, x), i \in I_{0}\right\}=\operatorname{rank}\left\{\nabla_{x} h_{i}\left(p^{0}, x^{0}\right), i \in I_{0}\right\} .
$$

Let rank $\left\{\nabla_{x} h_{i}\left(p^{0}, x^{0}\right), i \in I_{0}\right\}=k$. Then there exists indices $i_{1}, \ldots, i_{k} \subset I_{0}, i_{j} \neq i_{k}$ for $j \neq k$ such that $\nabla_{x} h_{i_{1}}\left(p^{0}, x^{0}\right), \ldots, \nabla_{x} h_{i_{k}}\left(p^{0}, x^{0}\right)$ are linearly independent. Denote $I_{0}^{\prime}=\left\{i_{1}, \ldots, i_{k}\right\}$. Then, by the continuity of gradients of $h_{i}, i=1, \ldots, n$ with respect to variable $x, \nabla_{x} h_{i}(\cdot, \cdot), i \in I_{0}^{\prime}$ are linearly independent in some neighbourhood of $\left(p^{0}, x^{0}\right)$.

In view of Proposition 3.6, RCRCQ implies that there exists a subset $I_{0}^{\prime} \subset I_{0}$ of indices of parametric system defined by the set-valued mapping $F$ such that

$$
\operatorname{rank}\left\{\nabla_{x} h_{i}(p, x), i \in I_{0}^{\prime}\right\}=\operatorname{rank}\left\{\nabla_{x} h_{i}\left(p^{0}, x^{0}\right), i \in I_{0}^{\prime}\right\}=\left|I_{0}^{\prime}\right|,
$$

for $(p, x)$ in some neighbourhood of $\left(p^{0}, x^{0}\right)$.

\section{RELAXED CONSTANT RANK CONDITION AND R-REgUlaRity}

It is known $[5,22]$ that the Mangasarian-Fromovitz constraint qualification (MFCQ) [23] for the set $F\left(p^{0}\right)$ at a point $x^{0} \in F\left(p^{0}\right)$ implies R-regularity of the set-valued mapping $F$ at $\left(p^{0}, x^{0}\right) \in g r F$.

We show that RCRCQ implies R-regularity of the set-valued mapping $F$. 
Theorem 4.1. Assume that 1) $F$ is Isc at $\left(p^{0}, x^{0}\right) \in \operatorname{gr} F$ relative to $\operatorname{dom} F$;

2) Fsatisfies $R C R C Q$ at $\left(p^{0}, x^{0}\right) \in \operatorname{gr} F$ relative to $\operatorname{dom} F \times \mathcal{H}$.

Then $F$ is $R$-regular at $\left(p,{ }^{0} x^{0}\right)$ relative to $\operatorname{dom} F$.

Proof. By Theorem 3.1, R-regularity of the mapping $F$ at $\left(p^{0}, x^{0}\right) \in g r F$ is equivalent to the fact that there exists a number $M>0$ such that for any sequences $p_{k} \rightarrow$ $p^{0}, p_{k} \in \operatorname{dom} F, v_{k} \rightarrow x^{0}, v_{k} \notin F\left(p_{k}\right)$, the inequality $\Lambda_{v}^{M}\left(p_{k}, x_{k}\right) \neq \emptyset$ holds for all $x_{k}=x\left(p_{k}, v_{k}\right) \in \Pi_{F\left(p_{k}\right)}\left(v_{k}\right)$ and $k$ sufficiently large.

On the contrary, suppose that there exist sequences $p_{k} \rightarrow p^{0}, v_{k} \rightarrow x^{0}, x_{k} \in F\left(p_{k}\right)$ such that $v_{k} \notin F\left(p_{k}\right), x_{k} \in \Pi_{F\left(p_{k}\right)}\left(v_{k}\right)$ and

$$
\operatorname{dist}\left(0, \Lambda_{v_{k}}\left(p_{k}, x_{k}\right)\right) \rightarrow+\infty .
$$

Due to the fact that $x^{0} \in \liminf _{p \rightarrow p^{0}} F(p)$, without loss of generality, we can assume that $F\left(p_{k}\right) \neq \emptyset$ for each $p_{k}, k=1, \ldots$, and for any $\hat{x}_{k} \in \Pi_{F\left(p_{k}\right)}\left(v_{k}\right)$ we have $\hat{x}_{k} \rightarrow x^{0}$. In consequence, $x_{k} \rightarrow x_{0}$.

As already noted, if RCRCQ holds at $\left(p^{0}, x^{0}\right)$, then RCRCQ holds also at all points close to $\left(p^{0}, x^{0}\right)$. Without loss of generality, we can assume that RCRCQ holds at all $\left(p_{k}, x_{k}\right), k=1,2, \ldots$. Consequently, by Lemma 3.1, $\Lambda_{v_{k}}\left(p_{k}, x_{k}\right) \neq \emptyset$ for all $k=1,2, \ldots$.

Without loss of generality, we can assume that $\left(p_{k}, v_{k}\right) \in V\left(p^{0}, x^{0}\right)$, where by RCRCQ, $V\left(p^{0}, x^{0}\right)$ is such that for any $J, I_{0} \subset J \subset I_{0} \cup I\left(p_{0}, x_{0}\right)$

$$
\operatorname{rank}\left\{\nabla_{x} h_{i}(p, x), i \in J\right\}=\operatorname{rank}\left\{\nabla_{x} h_{i}\left(p^{0}, x^{0}\right), i \in J\right\} \quad \forall(p, x) \in V\left(p^{0}, x^{0}\right) .
$$

By Lemma 2.1, $\Gamma\left(F\left(p^{k}\right), x^{k}\right)=T\left(F\left(p^{k}\right), x^{k}\right)$ and by the necessary optimality conditions for problem (3.2) ${ }^{1}$ we have

$$
v_{k}-x_{k}=\sum_{i \in I_{0} \cup I\left(p_{k}, x_{k}\right)} \hat{\lambda}_{i}^{k} \nabla_{x} h_{i}\left(p_{k}, x_{k}\right), \quad k=1, \ldots
$$

where $\hat{\lambda}_{i}^{k} \in \mathbb{R}, i \in I_{0}$ and $\hat{\lambda}_{i}^{k} \geq 0, i \in I\left(p_{k}, x_{k}\right), k=1, \ldots$ Recall that $I(p, x):=$ $\left\{i \in I \mid h_{i}(p, x)=0\right\}$ and $\hat{\lambda}_{i}^{k}, i \in I \cup I\left(p_{k}, x_{k}\right)$, are related to the set $\Lambda_{v_{k}}\left(p_{k}, x_{k}\right)$ via the relationship

$\frac{v_{k}-x_{k}}{\left\|v_{k}-x_{k}\right\|}=\sum_{i \in I_{0} \cup I\left(p_{k}, x_{k}\right)} \lambda_{i}^{k} \nabla_{x} h_{i}\left(p_{k}, x_{k}\right) \Longleftrightarrow v_{k}-x_{k}=\sum_{i \in I_{0} \cup I\left(p_{k}, x_{k}\right)} \hat{\lambda}_{i}^{k} \nabla_{x} h_{i}\left(p_{k}, x_{k}\right)$,

for some $\lambda^{k} \in \Lambda_{v_{k}}\left(p_{k}, x_{k}\right)$.

By Proposition 3.6, there exist neighbourhoods $V_{1}\left(p^{0}\right), V_{1}\left(x^{0}\right)$ and indices $I_{0}^{\prime} \subset I_{0}$, $\left|I_{0}^{\prime}\right|=\operatorname{rank}\left\{\nabla_{x} h_{i}\left(p_{0}, x_{0}\right) i \in I_{0}^{\prime}\right\}$ such that for all $(p, x) \in V_{1}\left(p^{0}\right) \times V_{1}\left(x^{0}\right)$ vectors $\nabla_{x} h_{i}(p, x), i \in I_{0}^{\prime}$ are linearly independent. Hence, without loss of generality we can assume that $\left(p_{k}, x_{k}\right) \in V_{1}\left(p^{0}\right) \times V_{1}\left(x^{0}\right)$, and for every $k=1, \ldots$, we have

$$
\sum_{i \in I_{0}} \hat{\lambda}_{i}^{k} \nabla_{x} h_{i}\left(p_{k}, x_{k}\right)=\sum_{i \in I_{0}^{\prime}} \check{\lambda}_{i}^{k} \nabla_{x} h_{i}\left(p_{k}, x_{k}\right)
$$

for some $\check{\lambda}_{i}^{k} \in \mathbb{R}, i \in I_{0}^{\prime}$.

\footnotetext{
${ }^{1}$ In the literature it is often assumed that Robinson constraint qualification holds (see for example [4]). However, it is enough to assume that the $T\left(F\left(p^{k}\right), x^{k}\right)$ coincides with feasible set to the linearized problem to (3.2) (see discussion after Lemma 3.7 of [4]).
} 
Hence we can rewrite (4.3) as follows

$$
v_{k}-x_{k}=\sum_{i \in I_{0}^{\prime}} \check{\lambda}_{i}^{k} \nabla_{x} h_{i}\left(p_{k}, x_{k}\right)+\sum_{i \in I\left(p_{k}, x_{k}\right)} \hat{\lambda}_{i}^{k} \nabla_{x} h_{i}\left(p_{k}, x_{k}\right),
$$

where $\nabla_{x} h_{i}\left(p_{k}, x_{k}\right), i \in I_{0}^{\prime}$, are linearly independent.

Passing to a subsequence, if necessary, we may assume that for all $k \in \mathbb{N}, I\left(p_{k}, x_{k}\right)$ is a fixed set, i.e., $I\left(p_{k}, x_{k}\right)=I^{0}$.

By [3, Lemma 2] we have that for any $k=1, \ldots$ there exists $I^{k} \subset I^{0}$ such that

$\sum_{i \in I_{0}^{\prime}} \check{\lambda}_{i}^{k} \nabla_{x} h_{i}\left(p_{k}, x_{k}\right)+\sum_{i \in I^{0}} \hat{\lambda}_{i}^{k} \nabla_{x} h_{i}\left(p_{k}, x_{k}\right)=\sum_{i \in I_{0}^{\prime}} \tilde{\lambda}_{i}^{k} \nabla_{x} h_{i}\left(p_{k}, x_{k}\right)+\sum_{i \in I^{k}} \tilde{\lambda}_{i}^{k} \nabla_{x} h_{i}\left(p_{k}, x_{k}\right)$,

where $\tilde{\lambda}_{i}^{k} \geq 0, i \in I^{k}$ and $\nabla_{x} h_{i}\left(p_{k}, x_{k}\right), i \in I_{0}^{\prime} \cup I^{k}$ are linearly independent. Hence we can rewrite (4.4) as

$$
v_{k}-x_{k}=\sum_{i \in I_{0}^{\prime}} \tilde{\lambda}_{i}^{k} \nabla_{x} h_{i}\left(p_{k}, x_{k}\right)+\sum_{i \in I^{k}} \tilde{\lambda}_{i}^{k} \nabla_{x} h_{i}\left(p_{k}, x_{k}\right) .
$$

Again, passing to a subsequence, if necessary, we may assume that $I^{k}=I^{\prime}$ is a fixed set.

Put $\lambda_{i}^{k}=\tilde{\lambda}_{i}^{k}\left\|v_{k}-p_{k}\right\|^{-1}, i \in I_{0}^{\prime} \cup I^{\prime}, k=1, \ldots$ and $\lambda_{i}^{k}=0, i \in I_{0} \cup I \backslash\left(I_{0}^{\prime} \cup I^{\prime}\right)$. Let us denote $\lambda^{k}=\left[\lambda_{i}^{k}\right]_{i \in I_{0}^{\prime} \cup I^{\prime}}$. We have $\lambda^{k} \in \Lambda_{v_{k}}\left(p_{k}, x_{k}\right)$ and, by (4.1), $\left\|\lambda^{k}\right\| \rightarrow+\infty$. Without loss of generality we may assume that $\lambda^{k}\left\|\lambda^{k}\right\|^{-1} \rightarrow \bar{\lambda}$. From (4.5) we have

$$
\frac{v_{k}-x_{k}}{\left\|v_{k}-x_{k}\right\|\left\|\lambda^{k}\right\|}=\sum_{i \in I_{0}^{\prime}} \frac{\lambda_{i}^{k}}{\left\|\lambda_{k}\right\|} \nabla_{x} h_{i}\left(p_{k}, x_{k}\right)+\sum_{i \in I^{k}} \frac{\lambda_{i}^{k}}{\left\|\lambda_{k}\right\|} \nabla_{x} h_{i}\left(p_{k}, x_{k}\right) .
$$

By passing to the limit in (4.6) we obtain

$$
0=\sum_{i \in I_{0}^{\prime} \cup I^{\prime}} \bar{\lambda}_{i} \nabla_{x} h_{i}\left(p^{0}, x^{0}\right), \bar{\lambda}_{i} \geq 0, i \in I^{\prime}
$$

where $\|\bar{\lambda}\|=1$. This contradicts the fact that for $k=1, \ldots$

$$
\begin{aligned}
& \operatorname{rank}\left\{\nabla_{x} h_{i}\left(p_{0}, x_{0}\right), i \in I_{0}^{\prime} \cup I^{\prime}\right\} \\
& =\operatorname{rank}\left\{\nabla_{x} h_{i}\left(p_{0}, x_{0}\right), i \in I_{0} \cup I^{\prime}\right\} \\
& =\operatorname{rank}\left\{\nabla_{x} h_{i}\left(p_{k}, x_{k}\right), i \in I_{0} \cup I^{\prime}\right\} \\
& =\operatorname{rank}\left\{\nabla_{x} h_{i}\left(p_{k}, x_{k}\right), i \in I_{0}^{\prime} \cup I^{\prime}\right\}=\left|I_{0}^{\prime} \cup I^{\prime}\right|,
\end{aligned}
$$

i.e. vectors $\nabla_{x} h_{i}\left(p_{0}, x_{0}\right), i \in I_{0}^{\prime} \cup I^{\prime}$ are linearly independent.

\section{LIPSCHITZ-LIKENESS OF $F$}

The following theorem provides the relationships between R-regularity and pseudoLipschitzness of the set-valued mapping $F$ defined on a normed space $\mathcal{G}$.

Theorem 5.1. Assume that $F$ is $R$-regular at a point $\left(p^{0}, x^{0}\right) \in \operatorname{gr} F$ relative to $\operatorname{dom} F$. Then $F$ is pseudo-Lipschitzian at this point relative to domF.

Proof. If $F$ is R-regular at a point $\left(p^{0}, x^{0}\right) \in \operatorname{gr} F$ relative to $\operatorname{dom} F$, this means that there are numbers $M>0, \delta>0, \varepsilon>0$ such that

$$
d(x, F(p)) \leq M \max \left\{0, h_{i}(p, x): i \in I,\left|h_{i}(p, x)\right|: i \in I_{0}\right\}
$$

for all $x \in V_{\varepsilon}\left(x^{0}\right)$ and all $p \in V_{\delta}\left(p^{0}\right) \cap \operatorname{dom} F$. 
Denote $l=\max \left\{l_{i} \mid i=1, \ldots, p\right\}$ where $l_{i}$ are Lipschitz constants for functions $h_{i}(p, x)$ on some set $V\left(p^{0}\right) \times V\left(x^{0}\right)$. Choose numbers $\delta$ and $\varepsilon$ such that $V_{\delta}\left(p^{0}\right) \subset$ $V\left(p^{0}\right), V_{\varepsilon}\left(x^{0}\right) \subset V\left(x^{0}\right)$. Then

$$
\begin{aligned}
& d\left(x^{0}, F(p)\right) \leq M \max \left\{0, h_{i}\left(p, x^{0}\right): i \in I,\left|h_{i}\left(p, x^{0}\right)\right|: i \in I_{0}\right\} \leq \\
& \leq M \max \left\{0, h_{i}\left(p, x^{0}\right)-h_{i}\left(p^{0}, x^{0}\right): i \in I,\left|h_{i}\left(p, x^{0}\right)-h_{i}\left(p^{0}, x^{0}\right)\right|: i \in I_{0}\right\} \leq l\left\|p-p^{0}\right\|
\end{aligned}
$$

for all $p \in V_{\delta}\left(p^{0}\right) \cap d o m F$.

This means that $F$ is Lipschitz lower continuous at $\left(p^{0}, x^{0}\right)$ relative to $\operatorname{dom} F$ and, consequently (see (2.1)), $F(p) \cap V_{\varepsilon}\left(x^{0}\right) \neq \emptyset$ for all $p \in V_{\eta}\left(p^{0}\right) \cap \operatorname{dom} F$, where $\eta<$ $\min \{\delta, \varepsilon / l\}$. Let $p, \tilde{p} \in V_{\eta}\left(p^{0}\right) \cap \operatorname{dom} F$ and let $\tilde{x} \in F(\tilde{p}) \cap V_{\varepsilon}\left(x^{0}\right)$. Then, from (5.1) follows

$$
\begin{aligned}
& d(\tilde{x}, F(p)) \leq M \max \left\{0, h_{i}(p, \tilde{x}): i \in I,\left|h_{i}(p, \tilde{x})\right|: i \in I_{0}\right\} \leq \\
& \leq M \max \left\{0, h_{i}(p, \tilde{x})-h_{i}(\tilde{p}, \tilde{x}): i \in I,\left|h_{i}(p, \tilde{x})-h_{i}(\tilde{p}, \tilde{x})\right|: i \in I_{0}\right\} \leq l\|p-\tilde{p}\| .
\end{aligned}
$$

The last inequality is equivalent to

$$
F(\tilde{p}) \cap V_{\varepsilon}\left(x^{0}\right) \subset F(p)+l\|p-\tilde{p}\| B \text { for all } p, \tilde{p} \in V_{\delta_{0}}\left(p^{0}\right) \cap d o m F .
$$

Remark 5.2. Let us note that in Theorem 5.1 we only need to assume that all the functions $f$ and $h_{i}, i=1, \ldots, n$ are locally Lipschitz continuous (may not be differentiable) near $\left(p^{0}, x^{0}\right) \in \operatorname{grF}$.

By Theorem 4.1 we obtain the following result

Theorem 5.3. Assume that 1) $F$ is $I s c$ at $\left(p^{0}, x^{0}\right) \in \operatorname{gr} F$ relative to $\operatorname{dom} F$;

2) Fsatisfies $R C R C Q$ at $\left(p^{0}, x^{0}\right) \in g r F$ relative to $\operatorname{dom} F \times \mathcal{H}$.

Then $F$ is pseudo-Lipschitzian at this point relative to $d o m F$.

Let us note that for some particular functions $h_{i}(\cdot, \cdot), i \in I \cup I_{0}$ Theorem 5.3 has been already proved in [3].

In the finite-dimensional setting, when both $\mathcal{H}$ and $\mathcal{G}$ are finite-dimensional spaces the results analogous to Theorem 5.3 can be obtained via properties of the optimal value function defined as $\varphi(p):=\inf \{f(p, x) \mid x \in F(p)\}$ and the solution set $S(p):=$ $\{x \in F(p) \mid f(p, x) \leq \varphi(p)\}$.

Definition 5.4. Let $p^{0} \in d o m F$. A mapping $F$ is locally bounded at $p^{0}$ if there exist a neighborhood $V\left(p^{0}\right)$ and a bounded set $Y_{0}$ such that $F(p) \subset Y_{0}$ for all $p \in V\left(p^{0}\right)$.

Theorem 5.5. Assume that 1) $F$ is locally bounded at $p^{0}$ and functions $f, h_{i}$ are Lipschitz continuous on a set $V\left(p^{0}\right) \times\left(Y_{0}+\varepsilon B\right)$, where $\varepsilon>2$ diam $Y_{0}$;

2) Fis $R$-regular at $\left(p^{0}, x^{0}\right) \in$ grF relative to $\operatorname{dom} F$.

Then $\varphi$ is Lipschitz continuous on some set $V\left(p^{0}\right) \cap \operatorname{dom} F$.

Proof. Let $l_{0}>0$ and $l_{i}>0$ be Lipschitz constants for $f$ and $h_{i}$ on a set $V\left(p^{0}\right) \times$ $\left(Y_{0}+\varepsilon B\right)$. Then due to Lemma 3.68 [22]

$\varphi(p)=\inf \left\{f(p, x)+\alpha d(x, F(p)) \mid x \in Y_{0}+2^{-1} \varepsilon B\right\}$ for all $p \in V\left(p^{0}\right)$ and any $\alpha>l_{0}$. 
Let us take any $p, \tilde{p} \in V\left(p^{0}\right)$ and $\tilde{x} \in S(\tilde{p})$. Then without loss of generality

$$
\begin{aligned}
& \varphi(p)-\varphi(\tilde{p}) \leq f(p, \tilde{x})+\alpha d(\tilde{x}, F(p))-f(\tilde{p}, \tilde{x}) \leq \\
& \leq f(p, \tilde{x})-f(\tilde{p}, \tilde{x})+\alpha M \max \left\{0, h_{i}(p, \tilde{x}): i \in I,\left|h_{i}(p, \tilde{x})\right|: i \in I_{0}\right\} \leq \\
& \leq l_{0}|p-\tilde{p}|+\alpha M \max \left\{0, h_{i}(p, \tilde{x})-h_{i}(\tilde{p}, \tilde{x}): i \in I,\left|h_{i}(p, \tilde{x})-h_{i}(\tilde{p}, \tilde{x})\right|: i \in I_{0}\right\} \leq \\
& \leq\left(l_{0}+\alpha M \max \left\{l_{i}: i \in I \cup I_{0}\right\}\right)\|p-\tilde{p}\| .
\end{aligned}
$$

Similarly, one can obtain $\varphi(\tilde{p})-\varphi(p) \leq\left(l_{0}+\alpha M \max \left\{l_{i}: i \in I \cup I_{0}\right\}\right)\|p-\tilde{p}\|$.

\section{Application to Bilevel Programming}

Consider a bilevel programming problem (BLPP):

$$
\begin{array}{ll}
\operatorname{minimize} & G(p, x), \\
\text { s.t. } & x \in S(p):=\arg \min \{f(p, x) \mid x \in F(p)\}, \\
& p \in P:=\left\{p \in \mathbb{R}^{n} \mid g_{j}(p) \leq 0 j \in J\right\},
\end{array}
$$

where $F(p)$ is defined in (1.1), $J=\{1, \ldots, l\}$, functions $G(p, x), g_{j}(p), f(p, x)$ and $h_{i}(p, x)$ are continuously differentiable (see e.g. monograph [7]).

The point $(p, x)$ is said to be a feasible point to the problem (BLPP) if $p \in P$, $x \in S(p)$. A feasible $\left(p^{0}, x^{0}\right)$ is called a solution (local solution) of the problem (BLPP) if $G\left(p^{0}, x^{0}\right) \leq G(p, x)$ for all feasible points $(p, x)$ (for all feasible points from some neighborhood of $\left.\left(p^{0}, x^{0}\right)\right)$.

The problem (BLPP) can be equivalently reformulated as the following one-level problem

$$
\begin{array}{ll}
\operatorname{minimize} & G(p, x) \\
\text { s.t. } & p \in P, x \in S(p)=\{x \in F(p) \mid f(p, x) \leq \varphi(p)\},
\end{array}
$$

where $\varphi(p)=\inf \{f(p, x) \mid x \in F(p)\}$ is the optimal value function of the lower-level problem. Main difficulty in solving problem (6.1) comes from the nonsmoothness of the value function $\varphi(p)$. Ye and Zhu [27] introduced the concept of partial calmness which allowed to move the nonsmooth constraint from the feasible set to the objective function.

Let $\left(p^{0}, x^{0}\right)$ be a feasible point of the problem (BLPP). The problem (BLPP) in the form (6.1) is called partially calm at $\left(p^{0}, x^{0}\right)$, if there exist a number $\mu>0$ and a neighborhood $V$ of the point $\left(p^{0}, x^{0}, 0\right)$ in $\mathbb{R}^{n} \times \mathbb{R}^{m} \times \mathbb{R}$ such that $G(p, x)-G\left(p^{0}, x^{0}\right)+$ $\mu|u| \geq 0$ for all $(p, x, u) \in V$ such that $p \in \mathcal{G}, x \in S(p), f(p, x)-\varphi(p)+u=0$.

In [27] it was proved that the problem (BLPP) in the form (6.1) is partial calm at its local solution $\left(p^{0}, x^{0}\right)$ if and only if there exists a number $\mu>0$ such that $\left(p^{0}, x^{0}\right)$ is a local solution of the partially penalized problem

$$
\begin{array}{ll}
\text { mimimize } & G(p, x)+\mu(f(p, x)-\varphi(p)), \\
\text { s.t. } & p \in P, x \in F(p) .
\end{array}
$$

Ye and Zhu [27] showed that the problem (BLPP) with a linear in $(p, x)$ lower-level problem is partially calm.

Let us define the set-valued mapping $S: p \rightrightarrows S(p)$ and consider the bilevel program (BLPP) under the following assumption:

(H1) $P \cap \operatorname{dom} S=P \cap \operatorname{dom} F$.

Denote $h_{0}(p, x):=f(p, x)-\varphi(p)$. Introduce the sets

$D:=\left\{(p, x) \mid h_{i}(p, x) \leq 0, i \in I, g_{j}(p) \leq 0, j \in J\right\}, C:=\left\{(p, x) \in D \mid h_{0}(p, x) \leq 0\right\}$. 
and $C(p):=\left\{x \in \mathbb{R}^{m} \mid(p, x) \in C\right\}$.

Theorem 6.1. Let $\left(p^{0}, x^{0}\right)$ be a local solution of problem (BLPP). Suppose that the mapping $S$ is R-regular at $\left(p^{0}, x^{0}\right)$ and the function $G$ is Lipschitz continuous on the set $D$ with Lipschitz constant $l_{0}>0$. Then there exists a number $\mu_{0}>0$ such that for any $\mu>\mu_{0}$ the point $\left(p^{0}, x^{0}\right)$ is a local solution to the problem

$$
\begin{array}{ll}
\operatorname{minimize} & G(p, x)+\mu(f(p, x)-\varphi(p)), \\
\text { s.t. } & (p, x) \in D .
\end{array}
$$

Proof. It follows from ( $\mathrm{H} 1)$ that $d(x, C(p))=d((p, x),\{p\} \times C(p)) \geq d((p, x), C)$ for all $(p, x) \in D$. In virtue of Proposition 2.4.3 of [6], for all $\alpha>l_{0}$, the point $\left(p^{0}, x^{0}\right)$ is a solution of the problem

$$
\begin{array}{ll}
\operatorname{minimize} & G(p, x)+\alpha d((p, x), C), \\
\text { s.t. } & (p, x) \in D .
\end{array}
$$

Then $d((p, x), C) \leq d(x, C(p))$ for all $(p, x) \in D$ and, therefore, $G(p, x)+\alpha d(x, C(p)) \geq$ $G\left(p^{0}, x^{0}\right)+\alpha d\left(x^{0}, C\left(p^{0}\right)\right)=G\left(p^{0}, x^{0}\right)$.

Since R-regularity for $S$ implies R-regularity for the set-valued $p \rightrightarrows C(p)$, there exists a neighbourhood $V\left(p^{0}, x^{0}\right)$ such that $G(p, x)+\alpha M \max \left\{0, h_{0}(p, x)\right\} \geq G\left(p^{0}, x^{0}\right)$ for all $(p, x) \in D \cap V\left(p^{0}, x^{0}\right)$. The last inequality is equivalent to the assertion of the theorem with $\mu_{0}=l_{0} M$.

\section{REFERENCES}

[1] Jean-Pierre Aubin. Lipschitz behavior of solutions to convex minimization problems. Math. Oper. Res., 9(1):87-111, 1984.

[2] Ewa M. Bednarczuk, Krzysztof W. Leśniewski, and Krzysztof E. Rutkowski. On tangent cone to systems of inequalities and equations under relaxed constant rank condition. arXiv e-prints, page arXiv:1905.05581, May 2019.

[3] Ewa M. Bednarczuk and Krzysztof E. Rutkowski. On Lipschitz-like property for polyhedral moving sets. arXiv e-prints, page arXiv:1811.05166, Nov 2018

[4] J. Frédéric Bonnans and Alexander Shapiro. Perturbation analysis of optimization problems. Springer Series in Operations Research. Springer-Verlag, New York, 2000.

[5] J. M. Borwein. Stability and regular points of inequality systems. J. Optim. Theory Appl., 48(1):952,1986

[6] Frank H. Clarke. Optimization and nonsmooth analysis. Canadian Mathematical Society Series of Monographs and Advanced Texts. John Wiley \& Sons, Inc., New York, 1983. A Wiley-Interscience Publication.

[7] Stephan Dempe. Foundations of bilevel programming, volume 61 of Nonconvex Optimization and its Applications. Kluwer Academic Publishers, Dordrecht, 2002.

[8] Andrei V. Dmitruk and Alexander Y. Kruger. Metric regularity and systems of generalized equations. J. Math. Anal. Appl., 342(2):864-873, 2008.

[9] Andrei V. Dmitruk and Alexander Y. Kruger. Extensions of metric regularity. Optimization, 58(5):561-584, 2009.

[10] A. L. Dontchev, M. Quincampoix, and N. Zlateva. Aubin criterion for metric regularity. J. Convex Anal., 13(2):281-297, 2006

[11] Asen L. Dontchev and R. Tyrrell Rockafellar. Implicit functions and solution mappings. Springer Series in Operations Research and Financial Engineering. Springer, New York, second edition, 2014. A view from variational analysis.

[12] V.V. Fedorov. Numerical maximin methods(russian book). Moscow, Izdatel'stvo Nauka, 1979. 280 p, In Russian, 1979.

[13] Lei Guo and Gui-Hua Lin. Notes on some constraint qualifications for mathematical programs with equilibrium constraints. J. Optim. Theory Appl., 156(3):600-616, 2013.

[14] Lei Guo, Jin Zhang, and Gui-Hua Lin. New Results on Constraint Qualifications for Nonlinear Extremum Problems and Extensions. Journal of Optimization Theory and Applications, 163(3):737754, Dec 2014. 
[15] René Henrion and Jiři V. Outrata. Calmness of constraint systems with applications. Math. Program., 104(2-3, Ser. B):437-464, 2005.

[16] A. D. loffe. Metric regularity and subdifferential calculus. Uspekhi Mat. Nauk, 55(3(333)):103$162,2000$.

[17] Alexander D. loffe. Variational analysis of regular mappings. Springer Monographs in Mathematics. Springer, Cham, 2017. Theory and applications.

[18] Robert Janin. Directional derivative of the marginal function in nonlinear programming. Math. Programming Stud., (21):110-126, 1984. Sensitivity, stability and parametric analysis.

[19] Diethard Klatte and Bernd Kummer. On calmness of the argmin mapping in parametric optimization problems. J. Optim. Theory Appl., 165(3):708-719, 2015.

[20] Alexander Y. Kruger. Error Bounds and Hölder Metric Subregularity. Set-Valued and Variational Analysis, 23(4):705-736, Dec 2015.

[21] Alexander Y. Kruger. Nonlinear metric subregularity. J. Optim. Theory Appl., 171(3):820-855, 2016.

[22] Bernd Luderer, Leonid Minchenko, and Tatyana Satsura. Multivalued analysis and nonlinear programming problems with perturbations, volume 66 of Nonconvex Optimization and its Applications. Kluwer Academic Publishers, Dordrecht, 2002.

[23] O. L. Mangasarian and S. Fromovitz. The Fritz John necessary optimality conditions in the presence of equality and inequality constraints. J. Math. Anal. Appl., 17:37-47, 1967.

[24] Leonid Minchenko and Sergey Stakhovski. On relaxed constant rank regularity condition in mathematical programming. Optimization, 60(4):429-440, 2011.

[25] Leonid Minchenko and Sergey Stakhovski. Parametric nonlinear programming problems under the relaxed constant rank condition. SIAM J. Optim., 21(1):314-332, 2011.

[26] R. Tyrrell Rockafellar and Roger J.-B. Wets. Variational analysis, volume 317 of Grundlehren der Mathematischen Wissenschaften [Fundamental Principles of Mathematical Sciences]. SpringerVerlag, Berlin, 1998.

[27] J. J. Ye and D. L. Zhu. Optimality conditions for bilevel programming problems. Optimization, 33(1):9-27, 1995. 